Photosynthetic reaction center variants made via genetic code expansion show Tyr at M210 tunes the initial electron transfer mechanism

Jared Bryce Weaver, ${ }^{1}$ Chi-Yun Lin, ${ }^{1}$ Kaitlyn M. Faries, ${ }^{2}$ Irimpan I. Mathews, ${ }^{3}$ Silvia Russi, ${ }^{3}$ Dewey Holten, ${ }^{2}$ Christine Kirmaier, ${ }^{2}$ Steven G. Boxer ${ }^{1, *}$

${ }^{1}$ Department of Chemistry, Stanford University, Stanford, California 94305, USA

${ }^{2}$ Department of Chemistry, Washington University, St. Louis, MO 63130-4889, USA

${ }^{3}$ Stanford Synchrotron Radiation Lightsource, 2575 Sand Hill Road, Menlo Park, California 94025, USA

*Correspondence to sboxer@stanford.edu 


\title{
Photosynthetic reaction center variants made via genetic code expansion show Tyr at M210 tunes the initial electron transfer mechanism
}

Jared Bryce Weaver, ${ }^{1}$ Chi-Yun Lin, ${ }^{1}$ Kaitlyn M. Faries, ${ }^{2}$ Irimpan I. Mathews, ${ }^{3}$ Silvia Russi, ${ }^{3}$ Dewey Holten, ${ }^{2}$ Christine Kirmaier, ${ }^{2}$ Steven G. Boxer ${ }^{1, *}$

${ }^{1}$ Department of Chemistry, Stanford University, Stanford, California 94305, USA

${ }^{2}$ Department of Chemistry, Washington University, St. Louis, MO 63130-4889, USA

${ }^{3}$ Stanford Synchrotron Radiation Lightsource, 2575 Sand Hill Road, Menlo Park, California 94025, USA

*Correspondence to sboxer@stanford.edu

\begin{abstract}
Photosynthetic reaction centers (RCs) from Rhodobacter sphaeroides were engineered to vary the electronic properties of a key tyrosine close to an essential electron transfer component (M210) via its replacement with site-specific genetically encoded noncanonical amino acid tyrosine analogs. High fidelity of noncanonical amino acid incorporation was verified with mass spectrometry and x-ray crystallography and demonstrated that RC variants exhibit no significant structural alterations relative to wild-type. Ultrafast transient absorption spectroscopy indicates the excited primary electron donor, $\mathrm{P}^{*}$, decays via a $\sim 4$ ps and a $\sim 20$ ps population to produce the charge-separated state $\mathrm{P}^{+} \mathrm{H}_{\mathrm{A}}{ }^{-}$in all variants. Global analysis indicates that in the $\sim 4$ ps population $\mathrm{P}^{+} \mathrm{H}_{\mathrm{A}}^{-}$forms through a 2-step process $\mathrm{P}^{*} \rightarrow \mathrm{P}^{+} \mathrm{B}_{\mathrm{A}}^{-}$ $\rightarrow \mathrm{P}^{+} \mathrm{H}_{\mathrm{A}}^{-}$, while in the $\sim 20$ ps population it forms via a 1-step $\mathrm{P}^{*} \rightarrow \mathrm{P}^{+} \mathrm{H}_{\mathrm{A}}^{-}$superexchange mechanism. The percentage of $\mathrm{P}^{*}$ population that decays via the superexchange route varies from $\sim 25 \%$ to $\sim 45 \%$ among variants while in wild-type this percentage is $\sim 15 \%$. Increases in the $\mathrm{P}^{*}$ population which decays via superexchange correlates with increases in free energy of the $\mathrm{P}^{+} \mathrm{B}_{\mathrm{A}}{ }^{-}$intermediate caused by a given M210 tyrosine analog. This was experimentally estimated through resonance Stark spectroscopy, redox titrations, and near-infrared absorption measurements. As the most energetically perturbative variant, 3-nitrotyrosine at M210 creates an $\sim 110 \mathrm{meV}$ increase in the free energy of $\mathrm{P}^{+} \mathrm{B}_{\mathrm{A}}{ }^{-}$along with a dramatic diminution of the $1030 \mathrm{~nm}$ transient absorption band indicative of $\mathrm{P}^{+} \mathrm{B}_{\mathrm{A}}^{-}$formation. Collectively this work indicates the tyrosine at M210 tunes the mechanism of primary electron transfer in the RC. reaction center | noncanonical amino acid | ultrafast transient absorption spectroscopy | Stark spectroscopy | superexchange | x-ray crystallography
\end{abstract}




\section{Significance}

Reaction centers (RCs) are critical to photosynthetic energy conversion. RCs in all characterized photosynthetic organisms contain two symmetrically arranged branches of chromophores and enable light-induced electron transfer with high yield. We fine-tune the properties of a key bacterial RC symmetry-breaking tyrosine via its replacement with noncanonical tyrosine analogs and determine kinetic outcomes. Results are interpreted through energetic characterization made possible by resonance Stark spectroscopy. Analysis indicates this tyrosine modulates the mechanism of the initial light-induced electron transfer, affording an alternative functional pathway that maintains the $\mathrm{RC}^{\prime} \mathrm{s}$ robust electron transfer. Modern molecular biology, ultrafast spectroscopy, crystallography, and energetic characterization enable the mechanistic model we describe. Our results deepen understanding of RC function and may have implications for other photocatalysts and enzymes.

Author contributions: J.B.W., C.-Y.L., D.H., C.K., and S.G.B. designed research; J.B.W., C.-Y.L., K.M.F., S.R., I.M., and C.K. performed experiments; J.B.W., C.-Y.L., K.M.F., I.M., D.H., and C.K. analyzed data; J.B.W, C.-Y.L., C.K., and S.G.B. wrote the paper.

\section{Introduction}

Photosynthetic reaction centers (RCs) are the integral membrane protein assemblies responsible for nearly all the solar energy capture maintaining our biosphere. In this study we focus on the initial electron transfer (ET) steps in bacterial RCs from Rhodobacter (R.) sphaeroides, a three-subunit (H, L, and M) $\sim 100 \mathrm{kDa}$ integral membrane protein complex. RCs in $R$. sphaeroides possess two branches of chromophores, the $A$ and $B$ (or $L$ and $M$ ) branches (Fig. 1A), and each possesses nearly identical chromophore composition, orientation, and distances. The protein secondary structure is pseudo-C2 symmetric, and the symmetry-related amino acids which differ are often structurally similar (Fig. 1A) (1). Despite this high structural symmetry, ET proceeds rapidly down only the A-branch of chromophores with near unity quantum yield $(2,3)$. Additionally, RC ET is remarkably robust, as few structurally verified single mutations that maintain RC chromophore composition and positioning significantly impact ET kinetics or yield $(1,4-11)$.

To understand RC ET asymmetry or unidirectionality and factors underlying its robust nature, a thorough understanding of the mechanism of ET is required. In the model largely accepted in the current literature $(1,12-14)$, A-branch ET is initiated by excitation of the excitonically coupled bacteriochlorophyll pair $\mathrm{P}$. The lowest singlet excited state $\mathrm{P}^{*}$ transfers an electron to the bacteriochlorophyll $B_{A}$ with a time constant of $\sim 3$ ps to form $\mathrm{P}^{+} B_{A}^{-}$. $B_{A}^{-}$subsequently transfers an 
electron to the bacteriopheophytin $\mathrm{H}_{\mathrm{A}}$ with a time constant of $1 \mathrm{ps}$, thus forming $\mathrm{P}^{+} \mathrm{H}_{\mathrm{A}}{ }^{-}$in a 2-step primary ET process, $\mathrm{P}^{*} \rightarrow \mathrm{P}^{+} \mathrm{B}_{\mathrm{A}}^{-} \rightarrow \mathrm{P}^{+} \mathrm{H}_{\mathrm{A}}^{-}(1,12,13)$. An alternative model for $\mathrm{ET}$ has been proposed in which $\mathrm{P}^{*}$ transfers an electron to $\mathrm{H}_{\mathrm{A}}$ directly through a superexchange mechanism, in which the $\mathrm{B}_{\mathrm{A}}$ chromophore mediates the electronic coupling between $\mathrm{P}^{*}$ and $\mathrm{H}_{\mathrm{A}}$. For wild-type (WT) RCs evidence favors 2-step ET at room temperature $(1,12,13,15,16)$. It has been previously proposed that minor degrees of superexchange occur in WT RCs, likely arising from or enhanced by the inherent distribution in the energies of $\mathrm{P}^{*}, \mathrm{P}^{+} \mathrm{B}_{\mathrm{A}}^{-}$, and $\mathrm{P}^{+} \mathrm{H}_{\mathrm{A}}{ }^{-}$caused by protein populations with slight variations in amino acid nuclear coordinates around chromophores (5,17-19), but this has been difficult to study experimentally $(1,12)$.

The ET mechanisms in RCs likely have their origins in the different energies of the various charge-separated states for the two branches relative to $\mathrm{P}^{*}$ and each other (Fig. 1B) (20-22) but it is difficult to determine these energetics either experimentally or theoretically $(1,23)$. Contributions of individual symmetry-breaking amino acid have been thoroughly studied $(1,15,24-28)$ and while the importance of certain amino acids has been ascertained, the roles of local protein-chromophore interactions are not always fully understood $(8,24,25)$. One highly examined residue has been the tyrosine at site $M 210$ ( $R C$ residue numbers are preceded by the protein subunit designation: $H, L$, or $M$ ) because it is a clear deviation in symmetry between $A$ - and B-branches (Fig. $1 A$ ), it is close to $B_{A}$, and it is the only one of 27 tyrosines which lacks a hydrogen bond acceptor. Theoretical studies indicates that the magnitude and orientation of the hydroxyl dipole of tyrosine M210 may play an important role in energetically stabilizing $\mathrm{P}^{+} \mathrm{B}_{\mathrm{A}}{ }^{-}(29,30)$. Indeed, previous efforts to change the orientation of this tyrosine's hydroxyl dipole significantly slowed ET (31). It is difficult, however, to subtly vary the electrostatic nature of this tyrosine using canonical mutagenesis without entirely removing the phenolic hydroxyl.

To perturb the effects of the tyrosine at M210 in WT protein, we used amber stop codon suppression (32-34) to site-specifically replace it with five noncanonical amino acids (ncAAs), each a tyrosine with a single electron donating or withdrawing meta-substituent (at the 3-position). We will refer to RC protein variants by acronyms for the amino acid incorporated at M210: 3-methyltyrosine (MeY), 3-nitrotyrosine $\left(\mathrm{NO}_{2} \mathrm{Y}\right)$, 3-chlorotyrosine (CIY), 3-bromotyrosine (BrY), and 3-iodotyrosine (IY) (Fig. 2, Fig. S1, Table S1). In this way, we engineered a series of RC variants with more systematic electrostatic ET perturbation at this important tyrosine, while minimally affecting other RC features. 


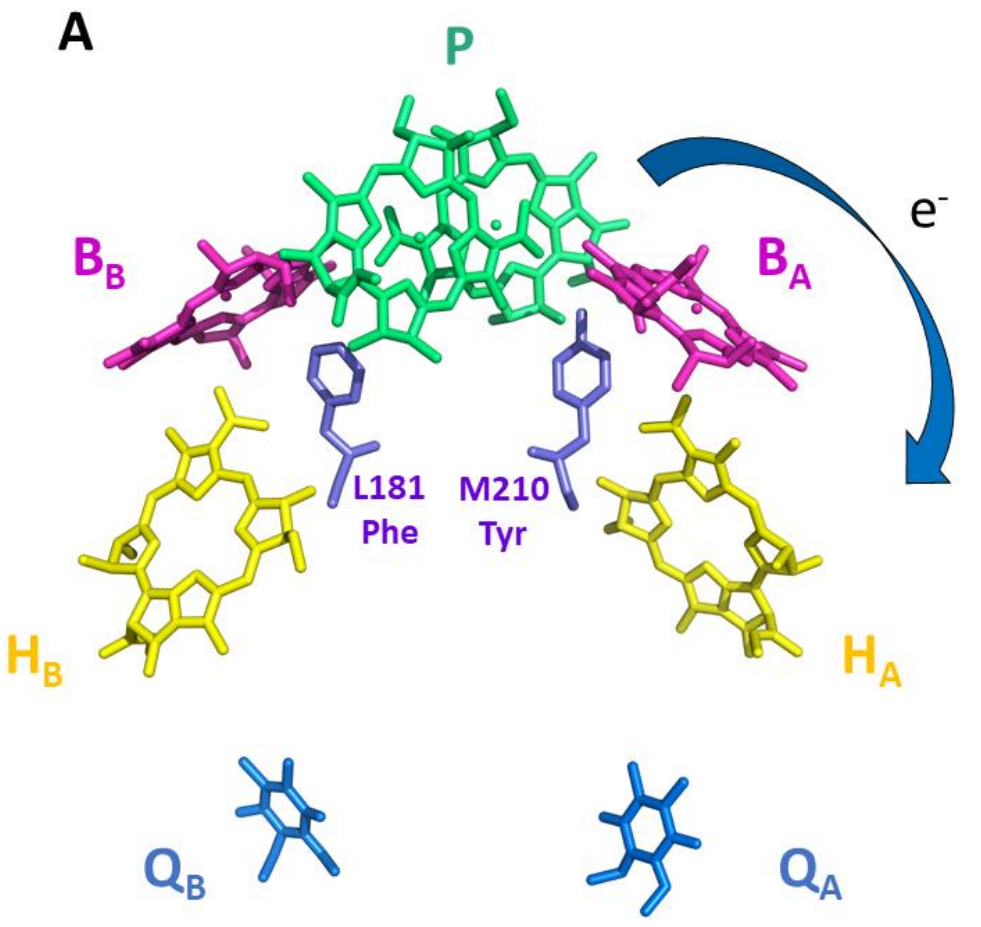

B

Fig. 1. RC chromophore arrangement and energetics. $(A)$ Chromophore arrangement in WT RCs (Protein Data Bank (PDB) ID: 2J8C; accessory carotenoid, chromophore phytyl tails, and quinone isoprenoid tails are removed here for clarity). Tyr at M210, the target in this work, and its symmetry-related residue Phe at L181 are shown in purple. The blue arrow indicates unidirectionality of ET down the A-branch. (B) Schematic free energy diagram of different charge-separated states in WT RCs, where $P *$ is $1.40 \mathrm{eV}$ above ground state and $\mathrm{P}^{+} \mathrm{H}_{\mathrm{A}}{ }^{-}$is $0.25 \mathrm{eV}$ below $\mathrm{P}^{*}$. The dashed magenta line and double headed arrow next to $\mathrm{P}^{+} \mathrm{B}_{\mathrm{A}}{ }^{-}$indicates the expected major effect of ncAA incorporation at $\mathrm{M} 210$ on the free energy of this state.

\section{Results}

Verification of ncAA Incorporation and Minimal Structural Perturbation. In previous work, we took an amber suppression system developed for halotyrosine (3-chlorotyrosine, 3-bromotyrosine, 3iodotyrosine) incorporation in E. coli (35) and transferred this system from E. coli to R. sphaeroides gene control, optimizing it for RC expression (36). Here, incorporation of 3-methyltyrosine (37) is also performed using this previously created system (SI Secs. S1.1 and S1.3-S1.4). We apply the same strategy used earlier (36) to a recently created amber suppression system (38) and genetically encode 3nitrotyrosine incorporation for the first time in $R$. sphaeroides (SI Secs. S1.1-S1.4). Liquid chromatography-mass spectrometry (LC-MS) was used to verify ncAA incorporation for $\mathrm{NO}_{2} \mathrm{Y}$ and MeY 
RCs (Figs. S2-S3). The mass shifts of the M-subunit peak correspond to the respective mass of the incorporated ncAAs (within a mass accuracy of \pm 1 Da for a $30 \mathrm{kDa}$ subunit) (Fig. S2), while the H- and Lsubunits remained identical to their WT mass as shown in previous work (36). This shows the general adaptability of our previous methods for genetic code expansion in $R$. sphaeroides.

$\mathrm{X}$-ray crystal structures of all variants were obtained at room temperature with resolutions comparable to those from previous room-temperature structures (39). These X-ray crystal structures (Fig. 2, Table S2, Fig. S4-S6) also verify ncAA incorporation at only site M210. All crystal structures possess additional electron density in the M210 tyrosine meta position (Fig. 2), which corresponds to the relevant ncAA incorporated with a phenolic position for the tyrosine side chain similar to WT (Fig. S5). $\mathrm{MeY}$ and $\mathrm{NO}_{2} \mathrm{Y}$ RCs have respective methyl- and nitro- substituents solely oriented towards $\mathrm{P}_{\mathrm{A}}$ while halotyrosine RCs display electron density (i.e., occupancy) that required modeling of two different halogen conformations, one oriented towards $\mathrm{P}_{\mathrm{A}}$ and the other towards the $\mathrm{Mg}^{2+}$ of $\mathrm{B}_{\mathrm{A}}$ (Fig. 2). While the halogen-substituted tyrosines likely have comparable electrostatic properties given their similar Hammett-parameters (40), $\mathrm{pK}_{\mathrm{a}}$ 's (41), and because they have been noted to affect processes involving charge transfer similarly (42), differences in their size may have led to the change in population oriented towards $\mathrm{P}_{\mathrm{A}}$ as opposed to $\mathrm{B}_{\mathrm{A}}$ since larger substituents are poorly accommodated by the smaller cavity next to $B_{A}$ (Fig. 2). For the $B_{A}$-oriented substituent, this leads to halogen-Mg ${ }^{2+}$ distances of $3.4 \AA$ (ClY), $3.4 \AA$ (BrY), and $2.9 \AA$ (IY). Dual halogen conformations for halotyrosine-containing proteins have been noted previously in other proteins $(37,42,43)$. Importantly, incorporation of all ncAAs at M210 in this study also proved to be minimally perturbative with respect to the remaining protein structure and chromophore positions and orientations; we found nearly identical interchromophoric distances and relative orientations to those present in the WT structure (PDB IDs: 2J8C and 1K6L; Figs. S4-S6, Table S3). Root-mean-square deviation (RMSD) is 0.229-0.261 for alignments to a room-temperature WT structure (1K6L) and is $0.333-0.367$ for alignment to a $77 \mathrm{~K}$ WT structure $(2 \mathrm{~J} 8 \mathrm{C})$. 


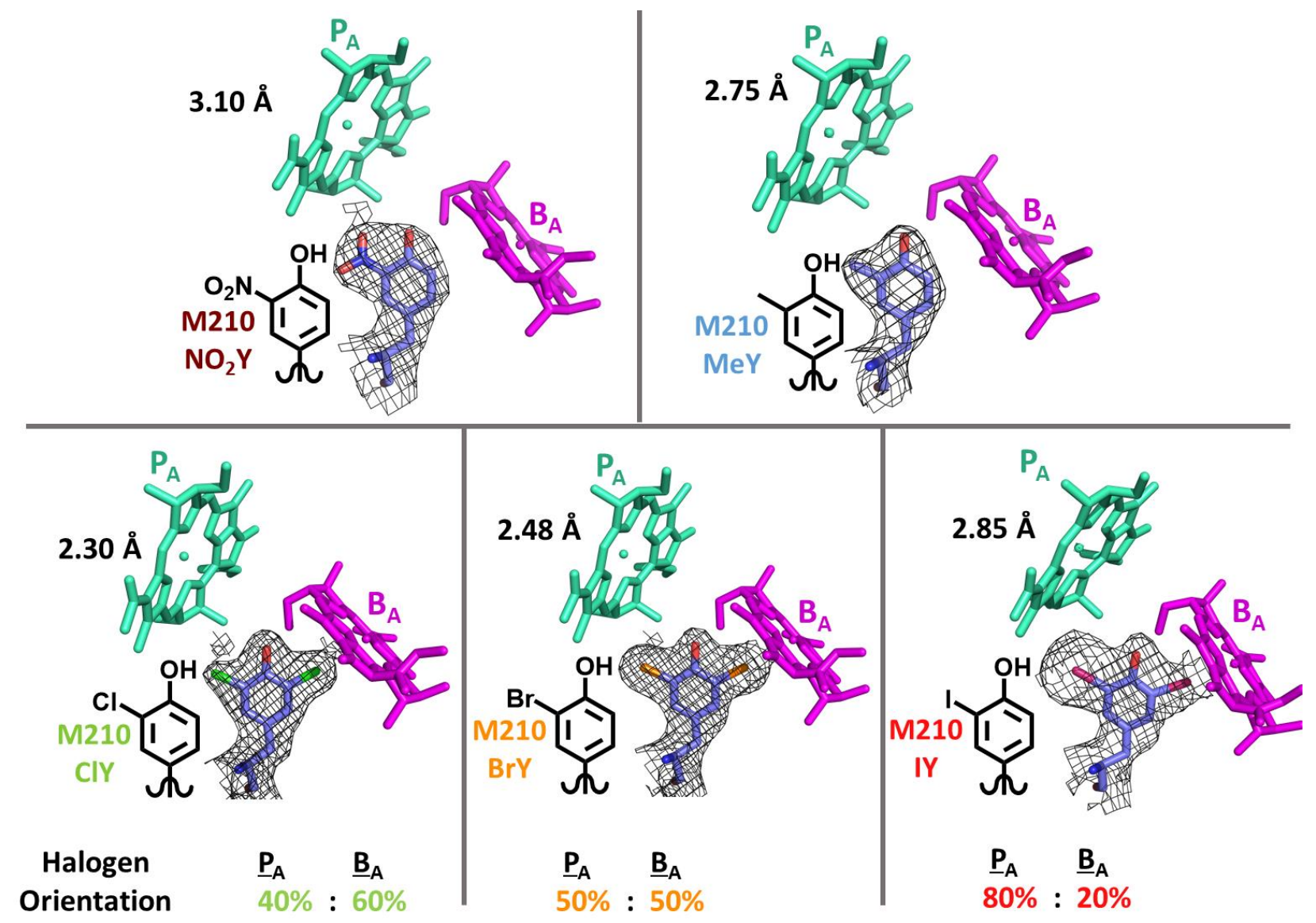

Fig. 2. $R C$ variants made in this study, where truncated $P_{A}$ and $B_{A}$ chromophores are depicted for each variant (in teal and magenta, respectively) and electron density maps from solved X-ray structures are shown $\left(2 F_{0}-F_{c}\right.$ contoured at $\left.1 \sigma\right)$ for tyrosine analogs at $M 210$. Halogen variants required two different tyrosine ring-conformers to model halogen substituent orientations with the contribution of each indicated, one with the halogen oriented towards $\mathrm{P}$ (only $\mathrm{P}_{\mathrm{A}}$ depicted above) and the other with halogen towards $B_{A}$. The resolution for each crystal structure is denoted in black next to the $P_{A}$ of each $R C$ variant (see SI Table S2).

Electron Transfer Kinetics. ET dynamics of the M210 variants were determined by transient absorption (TA) through a combination of (1) the assessment of raw spectra and single-wavelength kinetic fits (Fig. 3-5, Figs. S7-S38), (2) model-independent global analysis (Figs. S39-S55), and (3) model-dependent global analysis (Fig. 4, Figs. S56-S73). Results are summarized in Tables S4 and S5. TA kinetics at $924 \mathrm{~nm}$ and at $542 \mathrm{~nm}$ demonstrate that there is biexponential $\mathrm{P}^{*}$ decay and $\mathrm{P}^{+} \mathrm{H}_{\mathrm{A}}{ }^{-}$formation for all variants (Figs. S19 and S20). The biexponential $\mathrm{P}^{*}$ stimulated emission decay at $924 \mathrm{~nm}$ for WT and all variants reflects one $\mathrm{P}^{*}$ population that decays with a lifetime of $\sim 3 \mathrm{ps}$ and a second $\mathrm{P}^{*}$ population that decays 
with a lifetime of $\sim 20 \mathrm{ps}$ (Fig. S19 and Table S4). $\mathrm{P}^{+} \mathrm{H}_{\mathrm{A}}^{-}$formation detected at $542 \mathrm{~nm}$ has similar kinetics (Fig. S20 and Table S4). This biexponential $\mathrm{P}^{*}$ decay and $\mathrm{P}^{+} \mathrm{H}_{\mathrm{A}}{ }^{-}$formation has been noted previously in the literature for WT RCs $(6,10,15,18,19,44,45)$.

Global analysis of the TA data also supports biexponential $\mathrm{P}^{*}$ decay and $\mathrm{P}^{+} \mathrm{H}_{\mathrm{A}}^{-}$formation (Fig. 3, Table S4). A model-independent global fit returns decay-associated difference spectra (DADS), which are spectra composed of the pre-exponential coefficients at every wavelength for a given lifetime component in the fit. A DADS reflects the change in absorbance associated with a given lifetime $(46,47)$. While no DADS corresponds directly to one ET intermediate, it contains features indicative of the associated ET species. DADS spectral features and lifetimes guide a target model for global analysis that returns species-associated decay spectra (SADS) (see SI Section S3.2 for further discussion of SADS and DADS). DADS for all variants are remarkably similar; the same number of lifetimes are required to fit every variant except for $\mathrm{NO}_{2} \mathrm{Y}$ (Fig. 3), which did not require an $\sim 1$ ps component for a global fit. Spectral features for each time component were also very similar among variants. The $\sim 4 \mathrm{ps}$ and $\sim 1 \mathrm{ps}$ DADS (for all variants but $\mathrm{NO}_{2} \mathrm{Y}$ ) show features consistent with a 2-step ET model for $\mathrm{P}^{*} \rightarrow \mathrm{P}^{+} \mathrm{B}_{\mathrm{A}}{ }^{-} \rightarrow \mathrm{P}^{+} \mathrm{H}_{\mathrm{A}}{ }^{-}$. These features include those for $P^{+}(1250 \mathrm{~nm}), B_{A}^{-}(1030 \mathrm{~nm}), B_{A}$ and $P$ bleaching $(600 \mathrm{~nm})$, and $H_{A}$ bleaching (542 nm), all appropriately signed. In comparison, the 12-20 ps DADS showed features consistent with a superexchange ET model $\left(\mathrm{P}^{*} \rightarrow \mathrm{P}^{+} \mathrm{H}_{\mathrm{A}}^{-}\right)$, where $\mathrm{P}^{+}$and $\mathrm{H}_{\mathrm{A}}^{-}$features are present but the $1030 \mathrm{~nm}$ feature (associated with the $\mathrm{B}_{\mathrm{A}}{ }^{-}$anion absorption) is absent or significantly diminished, as noted previously (15). A greater proportion of the $P^{*}$ population that decays with the $\sim 20$ ps lifetime is present in all the variants than in WT (Fig. 3, Table S4). The $\sim 4 \mathrm{ps}$ DADS for the $\mathrm{NO}_{2} \mathrm{Y}$ variant does not possess a $1030 \mathrm{~nm}$ feature that would indicate $\mathrm{B}_{\mathrm{A}}{ }^{-}$production. Further DADS comparisons are given in Figs. S39-S55. 

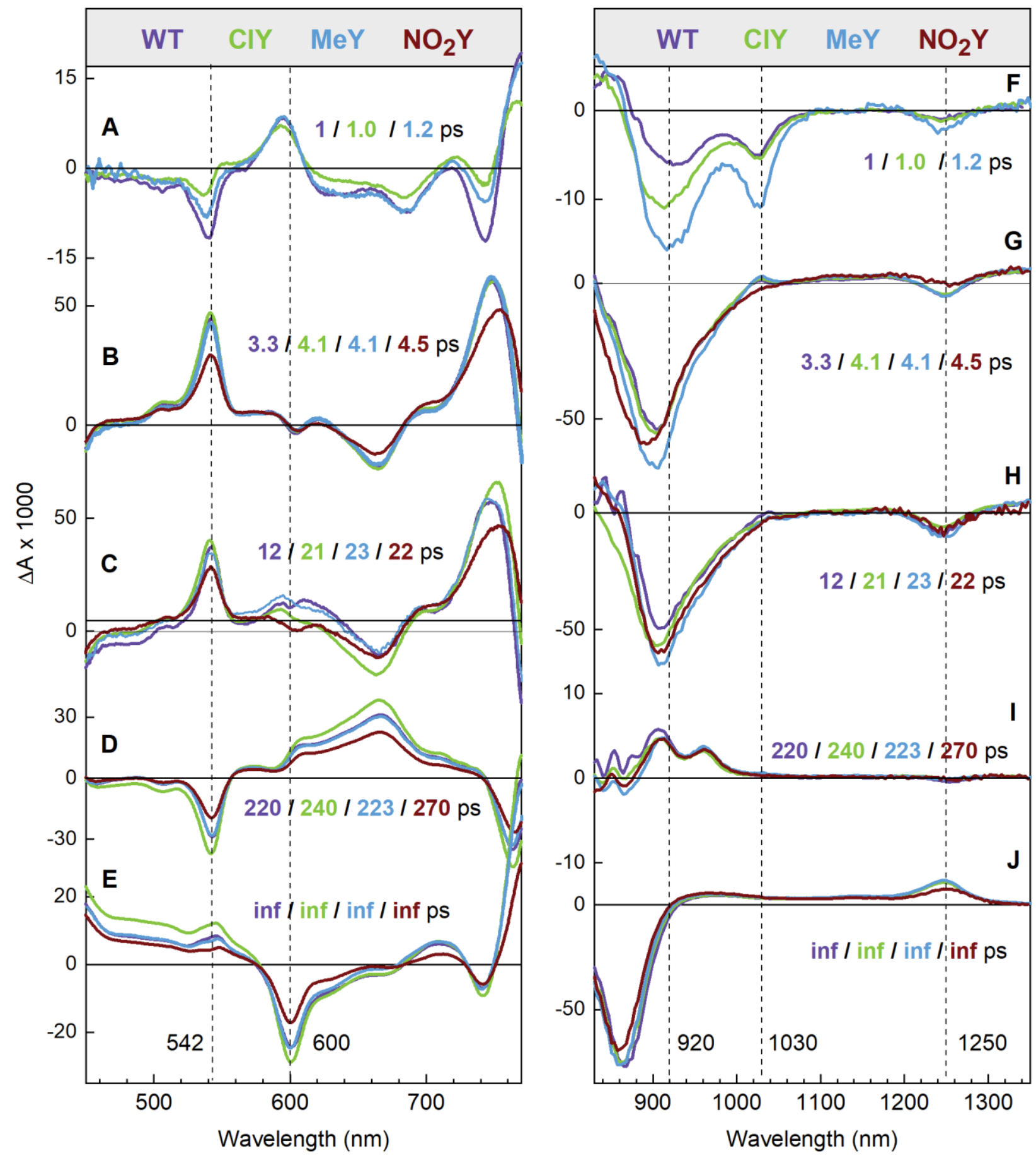

Fig. 3. Comparison of visible $(A-E)$ and near-IR (F-J) DADS for WT, CIY, MeY, and $\mathrm{NO}_{2} \mathrm{Y}$. DADS for BrY and IY are very similar to that of CIY and are given in Figs. S39-S43. Here "inf" refers to a component with an effectively infinite lifetime $\left(\sim 10^{8} \mathrm{ps}\right)$ to model charge transfer states that do not decay over the duration of the experiment $\left(10^{4} \mathrm{ps}\right)$. 
Based on the DADS features and single-wavelength kinetics discussed above, two $\mathrm{P}^{*}$ populations are present which give rise to $\mathrm{P}^{+} \mathrm{H}_{\mathrm{A}}{ }^{-}$via different mechanisms as opposed to simply reflecting the same process with differing rates (See SI Section S3.2 for details). For all the variants except $\mathrm{NO}_{2} \mathrm{Y}$, data were fit globally using a minimal target analysis model with one $\mathrm{P}^{*}$ population decaying via a 2-step ET process and the other via 1-step superexchange ET (Fig. 4A and S56A). Using this target model, we can reproduce differences in kinetics for each variant primarily by varying the relative amounts of the two $\mathrm{P}^{*}$ populations. As was seen from the DADS, the SADS also show a larger portion of the $\sim 20 \mathrm{ps} \mathrm{P}^{*}$ population in the variants relative to WT (Fig. 4B, Table S4). Additional effects include relatively small increases in $\mathrm{P}^{+} \mathrm{H}_{\mathrm{A}}{ }^{-}$lifetimes, similar to the effects of other mutations which are closer to $\mathrm{B}_{\mathrm{A}}$ than $\mathrm{H}_{\mathrm{A}}$ and also assumed nonperturbative on $\mathrm{H}_{\mathrm{A}}$ (Fig. 4B, Table S4) (48). Not needed in our target analysis (Fig. S56) is a $100-200$ ps component for $P^{*}$ internal conversion to the ground state $(27,49)$, consistent with all variants having a high quantum yield of $\mathrm{P}^{+} \mathrm{H}_{\mathrm{A}}{ }^{-}$formation similar to WT. Raw TA spectra also support this high quantum yield. At probe times where $\mathrm{P}^{+} \mathrm{H}_{\mathrm{A}}^{-}$is the dominant species present ( 25 ps) (Figs. S63, S65, S67, S69, S71, and S73) and in the $\mathrm{P}^{+} \mathrm{H}_{\mathrm{A}}^{-}$SADS (Fig. S60), the bleaching magnitudes at $542 \mathrm{~nm}\left(\mathrm{H}_{\mathrm{A}}^{-}\right.$) and $600 \mathrm{~nm}\left(\mathrm{P}^{*} / \mathrm{P}^{+}\right)$are roughly equal, consistent with the similar extinction coefficients of these two $\mathrm{H}_{\mathrm{A}}$ and $P$ features in the chromophore basis spectra $(50,51)$. The $600 \mathrm{~nm}$ bleach at $\sim 25 \mathrm{ps}$ is also similar in magnitude to the $600 \mathrm{~nm}$ bleach for $\mathrm{P}^{*}$ at $0.5 \mathrm{ps}$ (Panel A of Figs. S13-S18), again indicating high (essentially unity) conversion of $\mathrm{P}^{*} \rightarrow \mathrm{P}^{+} \mathrm{H}_{\mathrm{A}}{ }^{-}$in both populations.

Except for the $\mathrm{NO}_{2} \mathrm{Y}$ variant, the TA data clearly support a model in which 2-step and 1-step superexchange $\mathrm{ET}$ both occur. This is less certain in the $\mathrm{NO}_{2} \mathrm{Y}$ variant, for which the $1030 \mathrm{~nm} \mathrm{~B}_{\mathrm{A}}$ anion feature in the raw TA spectra and the DADS/SADS is absent or substantially diminished (Fig. 3, S18, S3638). This is most directly noted in the time evolution of the raw TA spectra in the 930-1100 nm window (Fig. 5, Figs. S21-S38). WT and CIY/BrY/IY/MeY variants display $\mathrm{B}_{\mathrm{A}}$ anion band formation centered at $1030 \mathrm{~nm}$, but $\mathrm{NO}_{2} \mathrm{Y}$ RCs show little to no integrated area for the $1030 \mathrm{~nm}$ transient feature above the baseline, which changes due to the background of $\mathrm{P}^{*}$ decay and growth of the $\mathrm{H}_{\mathrm{A}}^{-}$band at $\sim 960 \mathrm{~nm}$ (Fig. 5). The simplest explanation for the absent or significantly diminished $1030 \mathrm{~nm}$ band in the $\mathrm{NO}_{2} \mathrm{Y}$ variant is that $\mathrm{P}^{*}$ decays directly to $\mathrm{P}^{+} \mathrm{H}_{\mathrm{A}}{ }^{-}$via 1-step superexchange, without formation of $\mathrm{P}^{+} \mathrm{B}_{\mathrm{A}}{ }^{-}$as a discrete intermediate, in both $\mathrm{P}^{*}$ populations. Though the presence of a $\mathrm{P}^{*}$ population that undergoes fast $\sim 5 \mathrm{ps}$ superexchange ET, alongside the previously identified $\sim 12-20$ ps superexchange population $(15,19,52)$, has not been reported, we know of no reliable estimates that limit the rate of $\mathrm{P}^{*} \rightarrow \mathrm{P}^{+} \mathrm{H}_{\mathrm{A}}^{-}$ superexchange ET in RCs. Alternatively, it is possible that $\mathrm{P}^{+} \mathrm{B}_{\mathrm{A}}{ }^{-}$builds up to a much lesser extent in $\mathrm{NO}_{2} \mathrm{Y}$ RCs (compared to WT and the other variants) because the $\mathrm{P}^{+} \mathrm{B}_{\mathrm{A}}{ }^{-}$lifetime is much shorter. To account for 
the two possibilities, we modeled both scenarios for the $\mathrm{NO}_{2} \mathrm{Y}$ variant (Figs. S54-S56, Figs. S72-S73). Whichever model of ET is used, it is clear that primary ET in the $\mathrm{NO}_{2} \mathrm{Y}$ variant is affected more than primary ET in any other variant. Further mechanistic insight into $\mathrm{ET}$ in $\mathrm{NO}_{2} \mathrm{Y}$ and other RCs' ET requires information on how these RC variants change the free energy of $\mathrm{P}^{+} \mathrm{B}_{\mathrm{A}}^{-}$, as described in the following section (Energetic Characterization).

To be rigorous, it is important to note that superexchange ET involves a small degree of mixing of the $\mathrm{P}^{+} \mathrm{B}_{\mathrm{A}}{ }^{-}$virtual intermediate with the $\mathrm{P}^{*}$ reactant and $\mathrm{P}^{+} \mathrm{H}_{\mathrm{A}}{ }^{-}$product states when treated with firstorder perturbation theory (53). This mixing would imply that some minor degree of $B_{A}{ }^{-}$character could be manifest in spectral features, though significantly less than if $\mathrm{P}^{+} \mathrm{B}_{\mathrm{A}}{ }^{-}$were an actual intermediate. Computational insights into spectral contributions (e.g., at $1030 \mathrm{~nm}$ ) of a $\mathrm{P}^{+} \mathrm{B}_{\mathrm{A}}{ }^{-}$virtual intermediate in superexchange ET would be desirable.

As suggested by Niedringhaus et al. and others $(14,54)$ it is possible that the 2 -step vs superexchange mechanism dichotomy is too simplistic $(55,56)$. The difference in rates in the two $P^{*}$ populations in $\mathrm{NO}_{2} \mathrm{Y}$, which may both involve some form of superexchange $\mathrm{ET}$, also suggests that the usual formulation of the superexchange mechanism itself may be too simplistic. Electronic interactions exist not just between the pair of bacteriochlorophylls in P but also involve the other RC chromophores. Thus, the true mechanism may be an admixture of 2-step (i.e. sequential) ET and superexchange ET mechanisms, with the initial charge delocalized to varying extents over the $\mathrm{B}_{\mathrm{A}}$ and $\mathrm{H}_{\mathrm{A}}$ chromophores in the first ET intermediate depending on their relative energetics as discussed by Sumi et al. $(55,56)$. While we use limiting 2-step vs. superexchange models for tractability, further computational characterization of these more complex scenarios (55), and the limiting rates of superexchange ET in RCs are required to refine our analysis. 
A

2-Step Superexchange Population Population

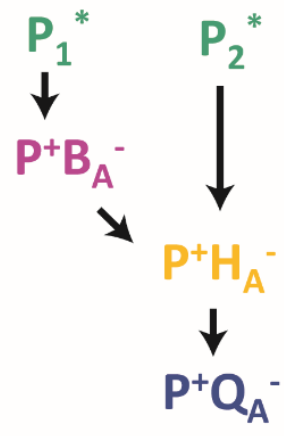

B

\begin{tabular}{|c|c|c|c|c|c|c|}
\hline $\mathbf{R C}$ & $\begin{array}{c}P_{1} * \\
\tau(p s)\end{array}$ & $\begin{array}{r}P_{2}{ }^{*} \\
\tau(p s)\end{array}$ & $\begin{array}{l}\mathrm{P}^{+} \mathrm{B}_{\mathrm{A}}{ }^{-} \\
\tau(\mathrm{ps})\end{array}$ & $\begin{array}{l}\mathrm{P}^{+} \mathrm{H}_{\mathrm{A}}{ }^{-} \\
\boldsymbol{\tau}(\mathbf{p s})\end{array}$ & $\begin{array}{l}\mathrm{P}_{1} * \\
\text { Pop. }\end{array}$ & $\begin{array}{l}P_{2} * \\
\text { Pop. }\end{array}$ \\
\hline WT & 2.6 & 20 & 1.5 & 220 & $86 \%$ & $14 \%$ \\
\hline MeY & 2.6 & 20 & 1.8 & 225 & $77 \%$ & $23 \%$ \\
\hline $\mathrm{ClY}$ & 2.5 & 20 & 1.7 & 240 & $54 \%$ & $46 \%$ \\
\hline BrY & 2.6 & 28 & 1.9 & 263 & $60 \%$ & $40 \%$ \\
\hline IY & 2.5 & 28 & 1.9 & 263 & $65 \%$ & $35 \%$ \\
\hline
\end{tabular}

Fig. 4. Target analysis model and fits. (A) Kinetic model implemented for target analysis for WT and all variants except $\mathrm{NO}_{2} \mathrm{Y}$ (see Fig. S56). (B) Species decay lifetimes and RC population (Pop.) decaying through a 2-step $\left(\mathrm{P}_{1}{ }^{*}\right)$ or superexchange $\left(\mathrm{P}_{2}{ }^{*}\right)$ ET pathway. While slight differences exist among RC variants for $\mathrm{P}_{1}{ }^{*}$ and $\mathrm{P}_{2}$ * decay time constants, data fit with a target analysis model with the same $\mathrm{P}^{*}$ decay lifetimes for all RC variants result in similar satisfactory SADS.
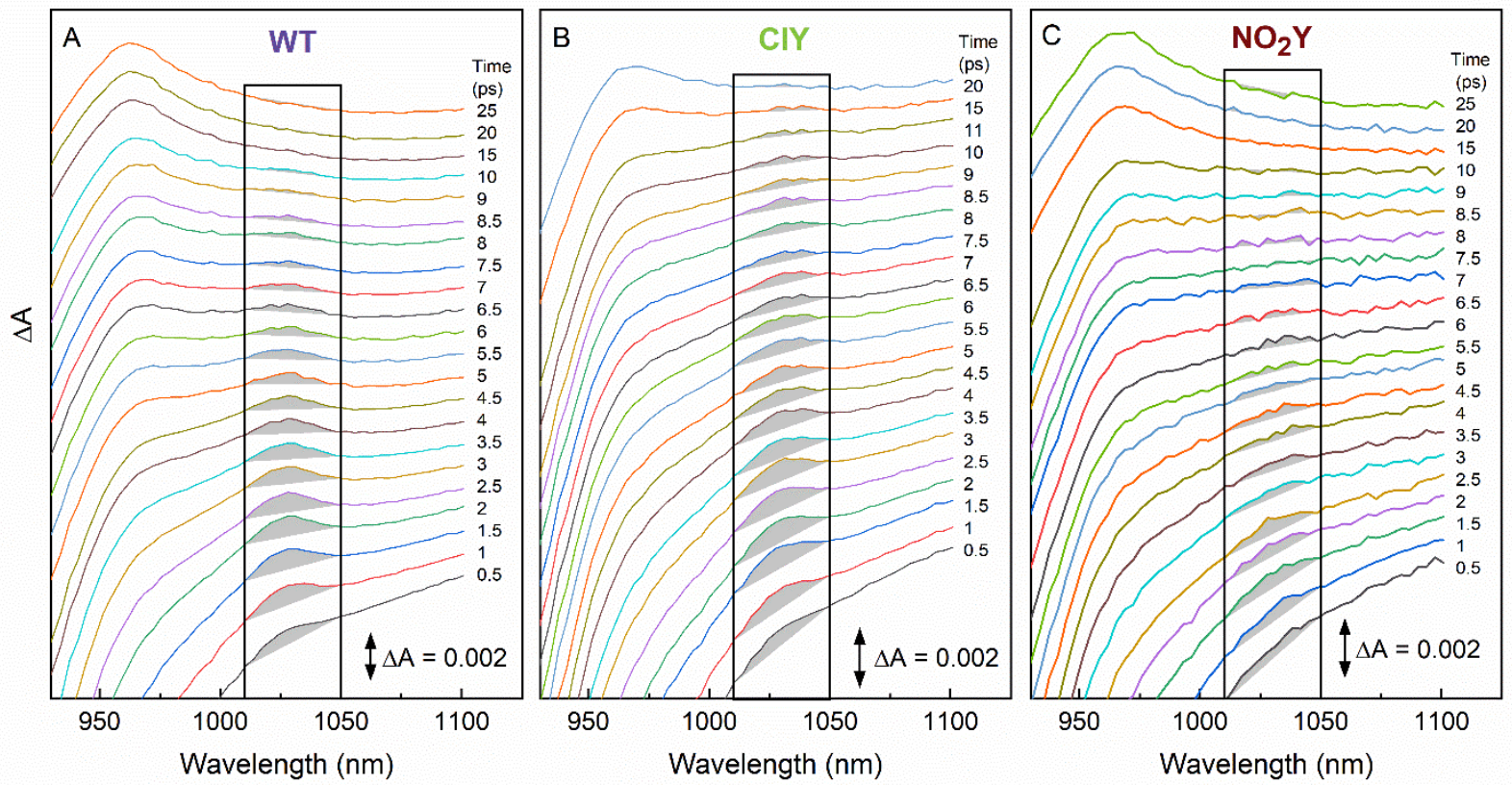

Fig. 5. Spectral evolution of the $930-1130 \mathrm{~nm}$ TA spectrum at early times for (A) WT, (B) ClY, and (C) NO2Y RCs (BrY/IY/MeY variants shown in Figs. S29, S32, and S35). The spectra are vertically offset from one another to best display the integrated area highlighted in gray $(1010-1050 \mathrm{~nm})$ of the $1030 \mathrm{~nm}$ band of P+BA- (see SI Sec. S3.6 for further detail). The gray integrated area for the $1030 \mathrm{~nm}$ feature was separated from $\mathrm{P}+\mathrm{HA}$ - background via an applied linear baseline. 
Energetic Characterization. To characterize the energetic perturbation of the tyrosine variants, we estimated the shift in $\mathrm{P}^{*} / \mathrm{P}^{+}$potential using $\mathrm{P}$ NIR absorption and $\mathrm{P} / \mathrm{P}^{+}$redox titrations and estimated the energetics of charge-separated intermediates using resonance Stark spectroscopy (Fig. 6). The roomtemperature NIR $Q_{y}$ absorbance maxima of $P$ shifted at most $15 \mathrm{~nm}\left(I Y, 179 \mathrm{~cm}^{-1}\right.$ or $22 \mathrm{meV}$ ) compared to WT (Fig. S74, Fig. S76). Redox titrations show P redox potentials shift by at most $26 \mathrm{mV}$ (IY, Figs. S75S76). When changes in both $\mathrm{P}$ absorption and $\mathrm{P}$ redox potential are combined as performed previously (10) (Fig. S76), RC variants at most disfavor P* oxidation by $18 \pm 5 \mathrm{meV}$ (MeY) relative to WT (Figs. 7 and S76B). These $P *$ oxidation energetic changes are minimal when compared to the $\sim 100-300$ meV free energy changes seen in certain mutants which target $P$ directly (10) and, given the proximity of residue M210 to $P$, are a surprising result, especially in the case of the $\mathrm{NO}_{2} \mathrm{Y} \mathrm{RC} \mathrm{(Fig.} \mathrm{2).}$

There is no direct way to obtain information on the $B_{A} / B_{A}{ }^{-}$reduction potential in situ. It is, however, possible to obtain information on the relative oxidation potentials of $B_{A}$ in these variants using resonance Stark effect (RSE) spectroscopy. $B_{A}{ }^{*}$ is coupled to $B_{A}{ }^{+} H_{A}{ }^{-}$whose energy is very sensitive to an applied electric field. We have described the RSE in detail in a series of papers (57-60) (see also SI Sections S5.1-5.3, Figs. S77-79), discovered over the course of measurements of higher order Stark effects for the RC and related pigments (61). Electronic Stark spectra ( $\Delta A$; field-on minus field-off absorbance) are typically obtained using lock-in detection at the second harmonic of the applied electric field modulation frequency, $\omega$, giving $\Delta A(2 \omega)$. For an isotropic immobilized sample, $\Delta A(2 \omega)$ is typically the second derivative of the absorption spectrum when the change in dipole moment between the ground and electronically excited states dominates the electrooptic parameters, as is the case for all the photosynthetic pigments in isolation (61). Data can also be extracted at higher even harmonics of the field modulation frequency, i.e. $4 \omega, 6 \omega$, etc.; $\Delta A(4 \omega)$ is typically the fourth derivative of the absorption (or second derivative of $\Delta A(2 \omega)$ ), $\Delta A(6 \omega)$ is the sixth derivative, and so on. Surprisingly, when this experiment is performed for $\mathrm{B}_{\mathrm{A}}{ }^{*}$ in the $\mathrm{RC}$ (but not for an isolated bacteriochlorophyll), a very different lineshape dominates the higher-order spectra $(57,62)$, as the conventional derivatives become very small. Through a series of experiments, this effect has been linked to the driving force for the $B_{A} * H_{A} \rightarrow$ $\mathrm{B}_{\mathrm{A}}{ }^{+} \mathrm{H}_{\mathrm{A}}{ }^{-}$reaction $\left(\Delta G_{1}\right)$ and a comprehensive theory describing the unusual but expected lineshapes of these RSEs has been developed (57-60). Since the Y(M210)F mutation dramatically affects the observed lineshape and amplitude (60), we expected other changes in the vicinity of $B_{A}$ could affect the RSE spectra and provide information on the energetics of this process. We then use information on changes in the $B_{A} / B_{A}{ }^{+}$potential to estimate effects on $B_{A} / B_{A}^{-}$, as described in detail below. 
As shown in Fig. 6A, it is immediately evident from the Stark spectra detected at the fourth harmonic of the modulation frequency, $\Delta A(4 \omega)$, that $M e Y$ is quite similar to $\mathrm{WT}$, that the halotyrosines show significantly reduced intensities compared to WT but are similar to each other, and that $\mathrm{NO}_{2} \mathrm{Y}$ has a very different lineshape (see Figs. S82-S88 for the sixth harmonic counterparts, $\Delta A(6 \omega)$ ). We seek to extract the change in driving force of an RC variant relative to $W T, \Delta \Delta G_{1}$, encoded in the change in $\delta$ (see Equation S22 in Section S5.3 for definition), from these lineshapes (Fig. 6B). As performed previously (60), this energetic determination assumes that WT and all RC variants have the same homogeneous and inhomogeneous line widths for $\mathrm{B}_{\mathrm{A}}$ (and for $\mathrm{B}_{\mathrm{B}}$ ) and identical reorganization energies for the pertinent charge transfer process (SI Sec. S5.3). Only one set of physically reasonable electron-transfer parameters (i.e., single fractional component fits) is required to reproduce the lineshapes and magnitudes of the Stark spectra for $\mathrm{WT}, \mathrm{MeY}$, and $\mathrm{NO}_{2} \mathrm{Y}$. As expected, the introduction of the weakly electron-donating methyl group barely perturbs $\Delta G_{1}$, while the strongly electron-withdrawing nitro group lowers the energetics of $B_{A}{ }^{*} H_{A} \rightarrow B_{A}{ }^{+} H_{A}{ }^{-}$by $\sim 100$ meV (Fig. 6B). The halotyrosine variants fail to conform to the one-fraction fit strategy; they give rise to unsatisfactory fits and/or require unphysically small charge-transfer distances ${ }^{\dagger}$ inferred from the diminished magnitude in Stark spectra (Figs. 6A and S85-S88) and inconsistent with the invariant interchromophoric distances between $B_{A}$ and $H_{A}$ observed in the x-ray structures across all variants (Table S3). Instead, a second fraction with a second set of electron transfer parameters is required. One reasonable origin for these two fractions in halotyrosine RCs is the two halogen orientations modeled in the crystal structures (Fig. 2); only one orientation is observed for $\mathrm{WT}, \mathrm{MeY}$, and $\mathrm{NO}_{2} \mathrm{Y}$, and a single-fraction fit to Stark spectra is sufficient for these samples. To deconvolve the underlying two fractions from the Stark spectra of halogenated RCs, we assume that the fraction that corresponds to the crystallographic population with the halogen atom pointing away from $B_{A}$ possesses WT-like energetics ( $\alpha$ fraction). This is justified by the fact that halogens typically do not substantially perturb the electronic properties of aromatic rings even when directly attached to the $\pi$-system being spectroscopically probed $(37,42)$, and because MeY, which is similarly weakly perturbative, leaves $\Delta G_{1}$ intact (Fig. 6B). By initializing fits with relative fractions from halogen occupancies in crystal structures, we reveal another fraction ( $\beta$ fraction; $F_{\beta}$ in Fig. $6 B$ ) that showed energetics more similar to those in the $\mathrm{NO}_{2} \mathrm{Y}$ counterpart $\left(\Delta \Delta G_{1} \sim-100 \mathrm{meV}\right.$; Fig. 6A, Figs. S81-S86). The Stark features of the underlying populations (see $\mathrm{WT}$ and $\mathrm{NO}_{2} \mathrm{Y}$ as the limiting cases) tend to cancel, leading to diminished magnitudes in $\Delta A(4 \omega)$ for halogenated species. In CIY, a larger $\beta$ fraction contribution leads to near complete Stark feature cancellation (Fig. 6A, green) while BrY and IY have a smaller contribution from the $\beta$ fraction and consequently less cancellation between Stark features from 
these two underlying fractions (Fig. 6A, orange and red). This degree of cancellation is largely consistent with the relative contributions of the two tyrosine rotamers as determined from crystal structures (Fig. 2). See SI Section S5.4 for a detailed analysis of the modeling of these spectra.

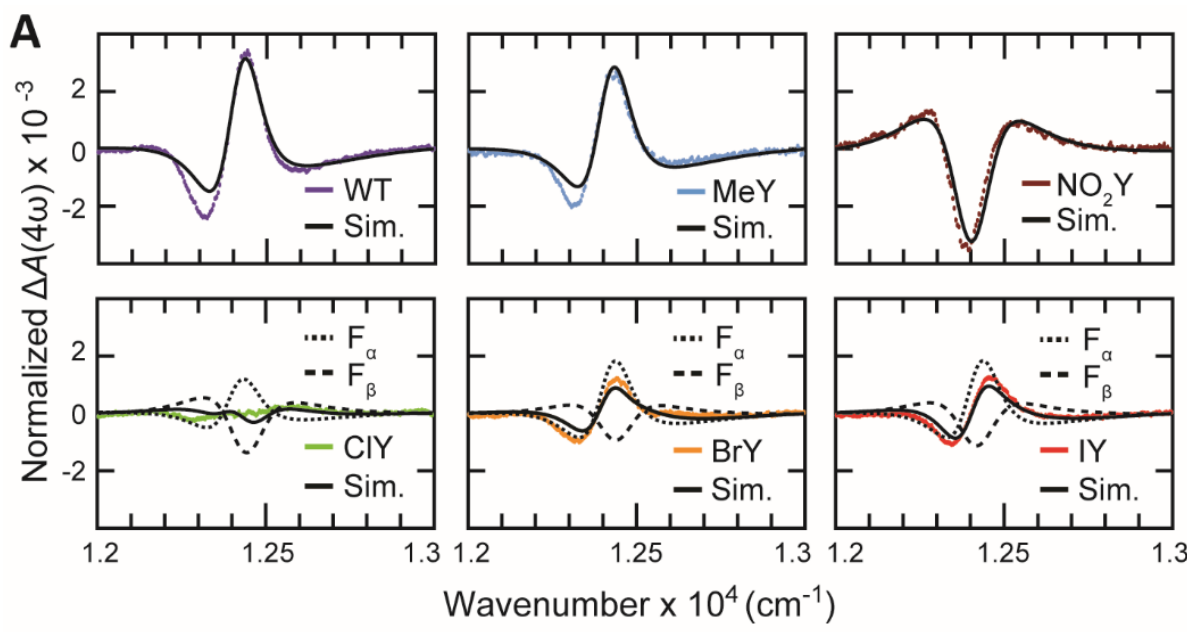

\begin{tabular}{|c|c|c|c|}
\hline $\mathrm{RC}$ & $\delta$ & $\begin{array}{r}\Delta \Delta G_{1} \\
(\mathrm{meV})\end{array}$ & $\begin{array}{l}F_{\beta} \\
(\%)\end{array}$ \\
\hline WT & -0.85 & 0 & 0 \\
\hline $\mathrm{MeY}$ & -0.85 & 0 & 0 \\
\hline $\mathrm{ClY}^{a}$ & -0.10 & -93 & 60 \\
\hline $\mathrm{Br}^{a}{ }^{a}$ & -0.05 & -99 & 40 \\
\hline$I Y^{a}$ & -0.05 & -99 & 40 \\
\hline $\mathrm{NO}_{2} \mathrm{Y}$ & -0.05 & -99 & 0 \\
\hline
\end{tabular}

Fig. 6. Resonance Stark spectra for $B_{A}$ and extracted energetics. $(A)$ Resonance Stark spectra $\Delta A$ determined for RC variants at the fourth harmonic $(4 \omega)$ of the external electric field modulation frequency $\omega$ (see SI Sections S5.1-5.4 for description and the corresponding $6 \omega$ spectra). Stark spectra are normalized to a field of $1 \mathrm{MV} / \mathrm{cm}$ (see Section S5.3 for further detail). (B) The values for the parameter, $\delta$, are extracted from the $\Delta A(4 \omega)$ and $\Delta A(6 \omega)$ Stark spectra in $(A)$ and SI Section 55.4 resp. and the change in $\Delta G_{1}$ relative to WT $\left(\Delta \Delta G_{1}\right)$ is determined from $\delta$ for the various RCs. Error in $\Delta \Delta G_{1}$ is $\sim 2 \mathrm{meV}$ and is due to fitting error. The energetic process associated with $\Delta \Delta G_{1}$ is depicted and labeled above the extracted Stark parameters. ${ }^{a}$ Halotyrosine Stark spectral fits require two fractions, which sum to 1 , and only the energetics for the $\beta$ fraction $\left(F_{\beta}\right)$ are shown above for halotyrosine RCs. The $\alpha$ fraction $\left(F_{\alpha}\right)$ for halotyrosine RCs is comparable to WT in energetics.

The resonance Stark data indicate tyrosine perturbation has a significant impact on $\Delta \Delta G_{1}$. Since tyrosine modification is significantly farther from the $\mathrm{H}_{A}$ chromophore than it is to $\mathrm{B}_{A}$ or $\mathrm{P}$ (Fig. 1A, Fig. S6A) and no structural changes are observed around $\mathrm{H}_{\mathrm{A}}$ (Fig. S6), we approximate the magnitude of $\Delta \Delta G_{1}$ to be due primarily to an impact on the energetics of $B_{A}$ oxidation. To better understand the origin of the observed transient absorption kinetics, we require information on $\mathrm{B}_{\mathrm{A}}$ reduction. We argue that $\Delta \Delta G_{1}$ should be roughly equal to the $-\Delta \Delta G$ for $B_{A} \rightarrow B_{A}{ }^{-}\left(\Delta \Delta G_{3}\right)$ under the two following approximations. (1) The $\mathrm{B}_{\mathrm{A}}{ }^{*}$ energy, $\mathrm{H}_{\mathrm{A}}$ reduction potential, and interchromophore coulombic interactions are not affected by tyrosine variant incorporation, as evidenced by the absence of structural changes and the insignificant energetic shifts in $B_{A}$ absorbance maxima (Figs. S74 and S76). (2) A general 
environmental effect of the mutations on the electronic structure of $B_{A}$ would impact the propensities for $\mathrm{B}_{\mathrm{A}}{ }^{+}$and $\mathrm{B}_{\mathrm{A}}{ }^{-}$formation in an equal and opposite fashion, i.e., any electronic polarization effect can be neglected. In this regard, since the $Q_{y}$ band of bacteriochlorins is dominated by the LUMO-HOMO gap (63), the HOMO and LUMO of $B_{A}$ appear to be shifted similarly by the tyrosine variants because the $Q_{y}$ band of $B_{A}$ (and $B_{B}$ ) at $800 \mathrm{~nm}$ is shifted at most $\sim 3 \mathrm{~nm}(\sim 4 \mathrm{meV}$ ) among the RC variants (Fig. S74). While these approximations may not be appropriate to characterize absolute energetics, they are reasonable to estimate changes in energetics relative to WT (see SI section S4.4 for further discussion of these approximations).

When we combine the changes in energetics of $\mathrm{P}^{*}$ oxidation and $\mathrm{B}_{\mathrm{A}}$ reduction, it is clear that the energetics of $\mathrm{P}^{*} \rightarrow \mathrm{P}^{+} \mathrm{B}_{\mathrm{A}}{ }^{-}$are significantly impacted in $\mathrm{NO}_{2} \mathrm{Y}$ and in one fraction $\left(\mathrm{F}_{\beta}\right)$ of the halotyrosine variants, increasing the energetics of $\mathrm{P}^{+} \mathrm{B}_{\mathrm{A}}^{-}$formation by over $100 \mathrm{meV}$ relative to $\mathrm{WT}\left(\Delta \Delta G_{4}\right.$ in Fig. 7). This is a significant change when considering $\mathrm{P}^{+} \mathrm{B}_{\mathrm{A}}^{-}$is estimated to be $\sim 70$ meV below $\mathrm{P}^{*}$ in energy in WT (Fig. 1B) $(14,38,55)$. In fact, in a recently published study performed at a high level of theory, Tamura et al. (22) indicate that $\mathrm{P}^{+} \mathrm{B}_{\mathrm{A}}{ }^{-}$is above $\mathrm{P}^{*}$ in energy in WT RCs, which, if correct, would mean (1) that the $\mathrm{P}^{*}$ $\rightarrow \mathrm{P}^{+} \mathrm{B}_{\mathrm{A}}^{-} \rightarrow \mathrm{P}^{+} \mathrm{H}_{\mathrm{A}}{ }^{-}$2-step process would not occur, and (2) that an $\sim 100$ meV energetic increase would be even more significant. Comparing $\mathrm{WT} \rightarrow \mathrm{MeY} \rightarrow$ halotyrosine $\mathrm{RCs}$, as the energetics of $\mathrm{P}^{+} \mathrm{B}_{\mathrm{A}}^{-}$formation $\left(\Delta \Delta G_{4}\right)$ increases (Fig. 7), so too does the amount of $\sim 20$ ps superexchange population observed in TA global analysis (Fig. 4B, Table S4). This would match the smaller energetic dependence of the rate constant for $\mathrm{P}^{+} \mathrm{B}_{\mathrm{A}}^{-}$formation in the superexchange mechanism compared to the rate constant in the two-step pathway (53), as the former mechanism is less disfavored than the latter by the increased $\mathrm{P}^{+} \mathrm{B}_{\mathrm{A}}{ }^{-}$ energy. For both models used for $\mathrm{NO}_{2} \mathrm{YET}$ (Fig. S56), there is also an increase in the $\sim 20$ ps $\mathrm{P}_{2}{ }^{*}$ population relative to WT (Tables S4 and Table S5, Fig. S72), similar to the other RC variants (Table S4). 


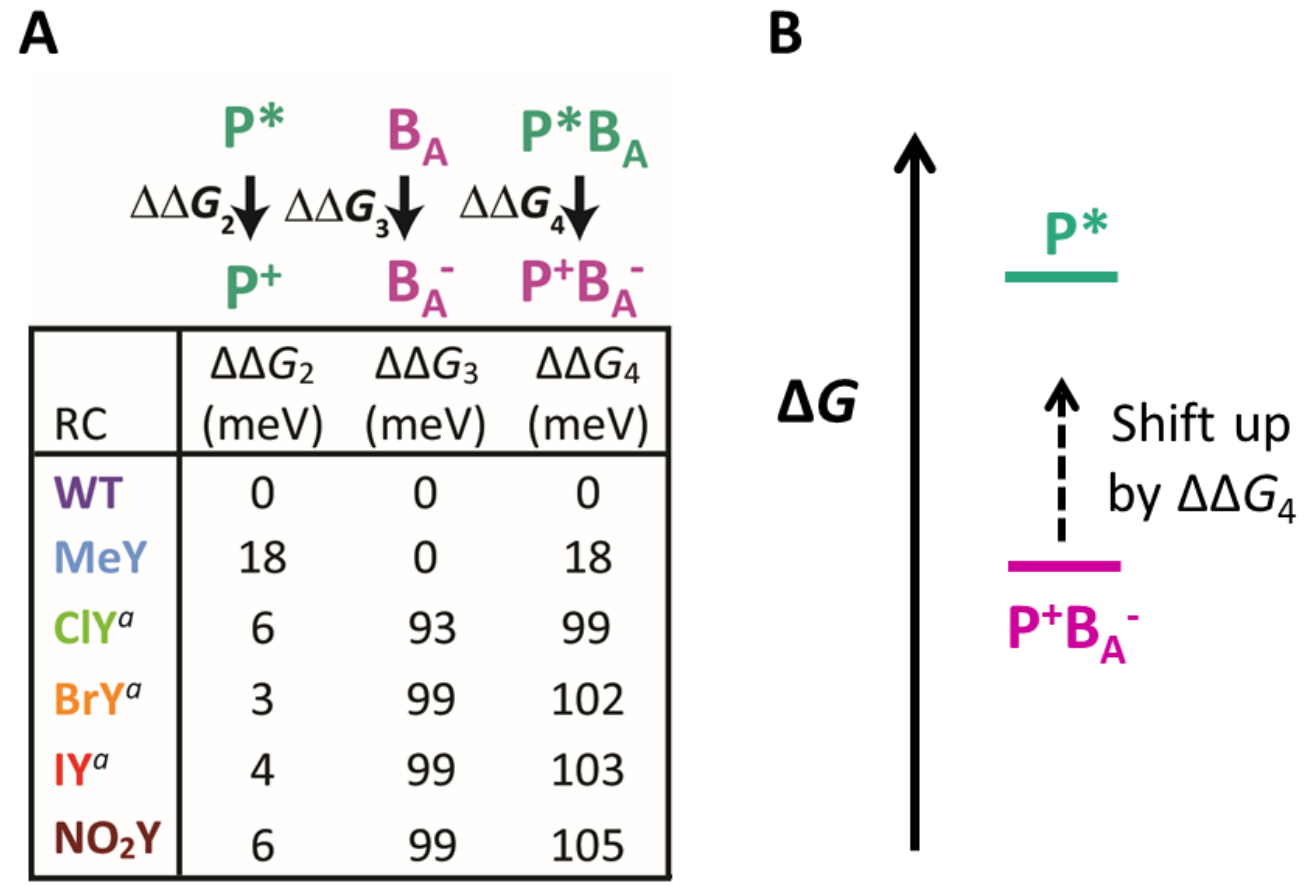

Fig. 7. (A) Relative change in energetics from WT for $\mathrm{P}^{*}$ oxidation $\left(\Delta \Delta G_{2}\right)$ and $\mathrm{B}_{\mathrm{A}}$ reduction $\left(\Delta \Delta G_{3}\right)$ from WT (see Fig. S76 for $\Delta \Delta G_{2}$ determination from NIR absorption and redox titration). We take $\Delta \Delta G_{2}+\Delta \Delta G_{3}$ $=\Delta \Delta G_{4}$ (where positive $\Delta \Delta G_{4}$ values are destabilizing). Energetic processes are depicted and labeled above each column. ${ }^{a}$ Energetics for halotyrosine variants are shown only for the resonance Stark population ( $\beta$ fraction) with non-WT $B_{A}$ redox energetics. $(B)$ Diagram depicting the impact of $\Delta \Delta G_{4}$, where the free energy of $\mathrm{P}^{+} B_{A}{ }^{-}$is raised by $\Delta \Delta G_{4}$.

Since $\mathrm{NO}_{2} \mathrm{Y} \mathrm{RCs}$ are like WT and other variants with regards to interchromophoric distances and other environmental features (Table S3), there is no obvious reason to explain why raising the free energy of the $\mathrm{P}^{+} \mathrm{B}_{\mathrm{A}}^{-}$state would cause its decay rate to increase dramatically. In fact, past studies indicate $\mathrm{P}^{+} \mathrm{B}_{\mathrm{A}}{ }^{-}$decay is already energetically barrierless in $\mathrm{WT}(5,66)$. The relative energetics of $\mathrm{H}_{\mathrm{A}}$ reduction are also unlikely to change significantly given the nitro group is significantly farther from $\mathrm{H}_{\mathrm{A}}$ than $B_{A}$ and $P$ (Fig. 1A, Fig. 2, Fig. S6A). Given the large increase in the free energy of $P^{+} B_{A}^{-}$in $N_{2} Y R C s$ in the context of barrierless ET, it does not seem likely that 2-step ET is occurring in $\mathrm{NO}_{2} \mathrm{Y} \mathrm{RCs}$ and instead the population normally undergoing 2-step ET likely transitions to a superexchange pathway. The P* population with a $\sim 5$ ps superexchange ET alongside the $\sim 20$ ps population more commonly identified as a superexchange population may reflect the relative energy denominators for electronic mixing in superexchange that depend on the relative energies for $\mathrm{P}^{+} \mathrm{B}_{\mathrm{A}}^{-}, \mathrm{P}^{+} \mathrm{H}_{\mathrm{A}}{ }^{-}$and $\mathrm{P}^{*}$. The absence of this change in mechanism (the predominant loss of a 2-step process) in halotyrosine RCs may be due to either or 
both of the following two effects: (1) only one fraction of halotyrosine RCs experience this large increase in energetics as evidenced by the two fractions observed from Stark spectroscopy and (2) crystallographic coordinates indicate that the fraction of RCs which appears correlated with changed energetics in Figs. 6 and $7\left(F_{\beta}\right)$ may have altered the electronic coupling(s) between $P^{*}$ and $B_{A}$ given the proximity of the halogens to the central $\mathrm{Mg}^{2+}$ of $\mathrm{B}_{\mathrm{A}}$ (Fig. 2).

\section{Discussion}

In this study we make a series of membrane protein complexes with site-specifically incorporated ncAAs. Within this series of pseudo-C2 symmetric proteins, we systematically vary one structural feature of an important symmetry-breaking amino acid, Tyr M210. In each tyrosine analogue, the tyrosine's electrostatic nature is altered with different electron-donating and -withdrawing substituents at the meta position, which modulates the tyrosine hydroxyl's interaction $(29,30,67)$ with the $B_{A}$ chromophore via induction. We then utilize three distinct techniques, $\mathrm{x}$-ray crystallography, TA spectroscopy, and resonance Stark spectroscopy, to determine structural information, ET kinetics, and the energetics relevant to primary ET. The collective information from these three distinct techniques helps to rationalize the consequences of our tyrosine analogue incorporation.

Structural analysis indicates none of these tyrosine variants caused measurable structural perturbation (i.e. nuclear displacement) of other protein residues, cofactors, or electron donors and acceptors (Figs. S4-S6, Table S3). Consequently, chromophore molecular orbital overlap(s) should be relatively unaffected by tyrosine perturbation and electronic coupling(s) and thus remain unchanged for the 2-step ET process. Halotyrosine variants may be the only exception to this given the proximity of the halogen to the central $\mathrm{Mg}^{2+}$ of $\mathrm{B}_{\mathrm{A}}$ in the crystallographic population in which the halogen is oriented towards $B_{A}$.

Stark spectroscopy indicates the energetics of ET involving $B_{A}$ are impacted significantly in halotyrosine ( $\beta$-fraction) and $\mathrm{NO}_{2} \mathrm{Y} \mathrm{RCs} \mathrm{(} 100 \mathrm{meV}$, Figs. $\left.6-7\right)$, while redox titrations and absorbance spectra indicate the effects on $\mathrm{P}^{*}$ oxidation are relatively minor ( $\leq 18 \mathrm{meV}$, Fig. 7). Only halotyrosine RCs require two conformers in crystallography and two energetic fractions for resonance Stark spectroscopy, but all RCs require two $\mathrm{P}^{*}$ populations to fit kinetic data; because of this observation, we only directly relate the resonance Stark fractions and the crystallographic conformations of halotyrosine RCs. Unlike in past studies $(4,10,19,48,68)$, our energetic approach is the first purely experimental estimate for the environmentally tuned energetics of $\mathrm{P}^{+} \mathrm{B}_{\mathrm{A}}{ }^{-}$formation. These estimations are at least qualitative and are treated in this study as semi-quantitative values. 
As these $\mathrm{RC}$ variants exhibit increasingly disfavored $\mathrm{P}^{+} \mathrm{B}_{\mathrm{A}}{ }^{-}$formation energetically (Figure 7 ), an increased proportion of the $\mathrm{P}^{*}$ population appear to decay by ET directly to $\mathrm{H}_{\mathrm{A}}$ via an $\sim 20$ ps superexchange mechanism (e.g. no $\mathrm{P}^{+} \mathrm{B}_{\mathrm{A}}{ }^{-}$intermediate) (Fig. 4) based on DADS/SADS and an ET competition model proposed previously (19). With our introduction of amber suppression into $R$. sphaeroides, we hope to site-specifically introduce ncAAs containing nitrile vibrational probes $(36,69-71)$ which can act as true ET spectators whose response will not be obfuscated by interchromophoric electronic coupling or electronic absorption shifts from the formation and decay of charged intermediates.

Despite the significant energetic impact of some ncAAs, no tyrosine modification made an order of magnitude impact on the overall rate $(5,10,31,72)$ or yield $(27)$ of $E T$, highlighting the robust nature of the RC's protein design (1,4-11). This small overall effect may be a consequence of this dualmechanism model, where the superexchange mechanism is acting as an alternative functional pathway for ET as previously proposed in the literature (19) through a lowered sensitivity to energetic changes of the $\mathrm{P}^{+} \mathrm{B}_{\mathrm{A}}{ }^{-}$intermediate. Without this additional pathway for ET, ET yield would likely decrease significantly following 3-nitrotyrosine introduction at M210 due to the increased amount of RC population possessing a $\mathrm{P}^{+} \mathrm{B}_{\mathrm{A}}{ }^{-}$state higher in free energy than $\mathrm{P}^{*}$. Clearly the tyrosine at $\mathrm{M} 210$ is not the only stabilizing factor for A-branch ET, as multiple protein features disfavor B-branch $E T(15,26,27,73)$, but it is important for tuning the mechanism to be one of 2-step ET in WT. This is highlighted by the $\mathrm{NO}_{2} \mathrm{YRC}$, where the increase in a slow $\mathrm{P} *$ population relative to $\mathrm{WT}$ is accompanied by the (near) absence of a $1030 \mathrm{~nm}$ feature associated with $B_{A}$ anion formation (Fig. 5, Figs. S21-S38) indicating a change in ET mechanism. Given the specificity and efficiency of 2-step ET in bacterial RCs, a tyrosine or phenol which interacts with an electron donor and acceptor, as does the tyrosine at M210, may be helpful to encourage charge transfer and develop or improve other synthetic catalysts (74-76) and enzymes $(77,78)$. Further research on this model for photoinduced charge transfer with tools such as amber stop codon suppression, will likely continue to enhance our understanding of how certain protein interactions are relevant to obtaining specific ET products, a key tenet of any catalysis and crucial to understanding ultrafast ET in the RC.

\section{Materials and Methods}

For a full description of methods for sample preparation, structural characterization via X-ray crystallography, transient absorption, spectroscopy, and redox titrations please see the Supporting Information. 


\section{Acknowledgements}

J.B.W. was supported by a Stanford Center for Molecular Analysis and Design Fellowship; C.-Y.L. was supported by a Kenneth and Nina Tai Stanford Graduate Fellowship and the Taiwanese Ministry of Education. This work was supported by a grant from the NSF Biophysics Program to S.G.B. (MCB1915727). K.M.F, D.H. and C.K. were supported by a grant from the U. S. Department of Energy, Office of Basic Energy Sciences (DE-CD0002036). We thank Prof. Ryan Mehl and Dr. Joseph Porter for providing the F4 and A7 gene sequences (SI Sections S1.1 and S1.2) as well as helpful discussion regarding 3nitrotyrosine incorporation in a new bacterial host. We thank the Vincent Coates Foundation Mass Spectrometry Laboratory, Stanford University Mass Spectrometry and particularly Theresa Laughlin for their support in performing the LC-MS in this study. We also thank Tom Carver in Stanford Nano Shared Facilities for depositing nickel on Stark windows.

\section{Footnotes}

'Short distances ranged from $7.0 \AA / f$ to $8.2 \AA / f$ as opposed to the $9.5 \AA / f$ to $13.1 \AA / f$ distances determined for RCs previously. Here $f$ is a local field factor which arises because the applied electric field is different from the field experienced by the chromophore and distances which are determined via resonance Stark spectroscopy contain this factor. The factor $f$ is greater than 1 and considered constant for different RCs (see SI Sections S5.2-S5.4 for further discussion).

\section{References}

1. W. Parson, A. Warshel, Mechanism of charge separation in Purple Bacterial Reaction Centers. The Purple Phototrophic Bacteria, 356-357 (2009).

2. C. A. Wraight, R. K. Clayton, The Absolute Quantum Efficiency of Bacteriochlorophyll Photooxidation in Reaction Centres of Rhodopseudomonos Spheroides. Biochimica et Biophysica Acta 333, 246-260 (1973).

3. R. K. Clayton, T. Yamamoto, Photochemical Quantum Efficiency and Absorption Spectra of Reaction Centers from Rhodopseudomonas Sphaeroides at Low Temperature. Photochemistry and Photobiology 24, 67-70 (1976).

4. L. M. P. Beekman, et al., Primary Electron Transfer in Membrane-Bound Reaction Centers with Mutations at the M210 Position. J. Phys. Chem. 100, 7256-7268 (1996).

5. V. Nagarajan, W. W. Parson, D. Davis, C. C. Schenck, Kinetics and Free Energy Gaps of ElectronTransfer Reactions in Rhodobacter sphaeroides Reaction Centers. Biochemistry 32, 12324-12336 (1993). 
6. U. Finkele, C. Lauterwasser, W. Zinth, K. Gray, D. Oesterhelt, Role of tyrosine M210 in the initial charge separation of reaction centers of Rhodobacter sphaeroides. Biochemistry 29, 8517-8521 (1990).

7. V. Nagarajan, W. W. Parson, D. Gaul, C. Schenck, Effect of specific mutations of tyrosine-(M)210 on the primary photosynthetic electron-transfer process in Rhodobacter sphaeroides. PNAS 87, 78887892 (1990).

8. E. T. Johnson, et al., Effects of Ionizable Residues on the Absorption Spectrum and Initial ElectronTransfer Kinetics in the Photosynthetic Reaction Center of Rhodobacter sphaeroides. Biochemistry 42, 13673-13683 (2003).

9. J. C. Williams, et al., Effects of mutations near the bacteriochlorophylls in reaction centers from Rhodobacter sphaeroides. Biochemistry 31, 11029-11037 (1992).

10. A. L. M. Haffa, et al., The Dependence of the Initial Electron-Transfer Rate on Driving Force in Rhodobacter sphaeroides Reaction Centers. J. Phys. Chem. B 106, 7376-7384 (2002).

11. S. Schenkl, et al., Selective perturbation of the second electron transfer step in mutant bacterial reaction centers. Biochimica et Biophysica Acta (BBA) - Bioenergetics 1554, 36-47 (2002).

12. W. Zinth, J. Wachtveitl, The First Picoseconds in Bacterial Photosynthesis - Ultrafast Electron Transfer for the Efficient Conversion of Light Energy. ChemPhysChem. 6, 871-880 (2005).

13. Y. Kakitani, A. Hou, Y. Miyasako, Y. Koyama, H. Nagae, Rates of the initial two steps of electron transfer in reaction centers from Rhodobacter sphaeroides as determined by singular-value decomposition followed by global fitting. Chemical Physics Letters 492, 142-149 (2010).

14. A. Niedringhaus, et al., Primary processes in the bacterial reaction center probed by twodimensional electronic spectroscopy. PNAS 115, 3563-3568 (2018).

15. P. D. Laible, et al., Switching sides-Reengineered primary charge separation in the bacterial photosynthetic reaction center. PNAS 117, 865-871 (2020).

16. A. G. Yakovlev, A. Ya. Shkuropatov, V. A. Shuvalov, Nuclear Wave Packet Motion between $\mathrm{P}^{*}$ and $\mathrm{P}^{+}$ $\mathrm{B}_{\mathrm{A}}{ }^{-}$Potential Surfaces with a Subsequent Electron Transfer to $\mathrm{H}_{\mathrm{A}}$ in Bacterial Reaction Centers at 90 K. Electron Transfer Pathway. Biochemistry 41, 14019-14027 (2002).

17. C. Kirmaier, D. Holten, Evidence that a distribution of bacterial reaction centers underlies the temperature and detection-wavelength dependence of the rates of the primary electron-transfer reactions. PNAS 87, 3552-3556 (1990).

18. Y. Jia, et al., Primary Charge Separation in Mutant Reaction Centers of Rhodobacter capsulatus. J Phys. Chem. 97, 13180-13191 (1993).

19. M. Bixon, J. Jortner, M. E. Michel-Beyerle, A kinetic analysis of the primary charge separation in bacterial photosynthesis. Energy gaps and static heterogeneity. Chemical Physics 197, 389-404 (1995). 
20. M. R. Gunner, A. Nicholls, B. Honig, Electrostatic Potentials in Rhodopseudomonas viridis Reaction Centers: Implications for the Driving Force and Directionality of Electron Transfer ${ }^{\dagger}$. J. Phys. Chem. 100, 4277-4291 (1996).

21. W. Parson, Z.-T. Chub, A. Warshel, Electrostatic control of charge separation in bacterial photosynthesis. Biochimica et Biophysica Acta (BBA) - Bioenergetics 1017, 251-272 (1990).

22. H. Tamura, K. Saito, H. Ishikita, Acquirement of water-splitting ability and alteration of the chargeseparation mechanism in photosynthetic reaction centers. PNAS 117, 16373-16382 (2020).

23. M. Richter, B. P. Fingerhut, Coupled excitation energy and charge transfer dynamics in reaction centre inspired model systems. Faraday Discuss. 216, 72-93 (2019).

24. M. Saggu, S. D. Fried, S. G. Boxer, Local and Global Electric Field Asymmetry in Photosynthetic Reaction Centers. J Phys Chem B (2019) https:/doi.org/10.1021/acs.jpcb.8b11458.

25. S. J. Robles, J. Breton, D. C. Youvan, Partial symmetrization of the photosynthetic reaction center. Science 248, 1402-1405 (1990).

26. N. P. Dylla, et al., Species differences in unlocking B-side electron transfer in bacterial reaction centers. FEBS Letters 590, 2515-2526 (2016).

27. J. I. Chuang, S. G. Boxer, D. Holten, C. Kirmaier, High Yield of M-Side Electron Transfer in Mutants of Rhodobacter capsulatus Reaction Centers Lacking the L-Side Bacteriopheophytin. Biochemistry 45, 3845-3851 (2006).

28. E. J. Bylina, C. Kirmaier, L. McDowell, D. Holten, D. C. Youvan, Influence of an amino-acid residue on the optical properties and electron transfer dynamics of a photosynthetic reaction centre complex. Nature 336, 182-184 (1988).

29. R. Alden, W. Parson, Z. Chu, A. Warshel, Orientation of the OH Dipole of Tyrosine (M) 210 and Its Effect on Electrostatic Energies in Photosynthetic Bacterial Reaction Centers. J Phys. Chem. 100, 16761-16770 (1996).

30. K. Kawashima, H. Ishikita, Energetic insights into two electron transfer pathways in light-driven energy-converting enzymes. Chem. Sci. 9, 4083-4092 (2018).

31. M. Saggu, et al., Putative hydrogen bond to tyrosine M208 in photosynthetic reaction centers from Rhodobacter capsulatus significantly slows primary charge separation. J. Phys. Chem. B 118, 672132 (2014).

32. A. Dumas, L. Lercher, C. D. Spicer, B. G. Davis, Designing logical codon reassignment - Expanding the chemistry in biology. Chem. Sci. 6, 50-69 (2015).

33. I. Drienovská, G. Roelfes, Expanding the enzyme universe with genetically encoded unnatural amino acids. Nat. Catal. 3, 193-202 (2020).

34. D. D. Young, P. G. Schultz, Playing with the Molecules of Life. ACS Chem. Biol. 13, 854-870 (2018). 
35. H. S. Jang, et al., Efficient Site-Specific Prokaryotic and Eukaryotic Incorporation of Halotyrosine Amino Acids into Proteins. ACS Chem. Biol. 15, 562-574 (2020).

36. J. B. Weaver, S. G. Boxer, Genetic Code Expansion in Rhodobacter sphaeroides to Incorporate Noncanonical Amino Acids into Photosynthetic Reaction Centers. ACS Synth. Biol. 7, 1618-1628 (2018).

37. C.-Y. Lin, M. G. Romei, L. M. Oltrogge, I. I. Mathews, S. G. Boxer, Unified Model for Photophysical and Electro-Optical Properties of Green Fluorescent Proteins. J. Am. Chem. Soc. 141, 15250-15265 (2019).

38. J. J. Porter, et al., Genetically Encoded Protein Tyrosine Nitration in Mammalian Cells. ACS Chem. Biol. 14, 1328-1336 (2019).

39. P. R. Pokkuluri, et al., The Structure of a Mutant Photosynthetic Reaction Center Shows Unexpected Changes in Main Chain Orientations and Quinone Position ${ }^{\dagger}$. Biochemistry 41, 5998-6007 (2002).

40. L. P. Hammett, The Effect of Structure upon the Reactions of Organic Compounds. Benzene Derivatives. J. Am. Chem. Soc. 59, 96-103 (1937).

41. John R. Rumble, ed., "Physical Constants of Organic Compounds", in CRC Handbook of Chemistry and Physics, 101st (Internet Version 2020), (CRC Press/Taylor \& Francis).

42. M. G. Romei, C.-Y. Lin, I. I. Mathews, S. G. Boxer, Electrostatic control of photoisomerization pathways in proteins. Science 367, 76-79 (2020).

43. Y. Wu, S. D. Fried, S. G. Boxer, Dissecting Proton Delocalization in an Enzyme's Hydrogen Bond Network with Unnatural Amino Acids. Biochemistry 54, 7110-7119 (2015).

44. P. Hamm, et al., Subpicosecond emission studies of bacterial reaction centers. Biochimica et Biophysica Acta (BBA) - Bioenergetics 1142, 99-105 (1993).

45. J. Zhu, I. H. M. van Stokkum, L. Paparelli, M. R. Jones, M. L. Groot, Early Bacteriopheophytin Reduction in Charge Separation in Reaction Centers of Rhodobacter sphaeroides. Biophysical Journal 104, 2493-2502 (2013).

46. I. H. M. van Stokkum, D. S. Larsen, R. van Grondelle, Global and target analysis of time-resolved spectra. Biochimica et Biophysica Acta (BBA) - Bioenergetics 1657, 82-104 (2004).

47. K. M. Faries, et al., Manipulating the Energetics and Rates of Electron Transfer in Rhodobacter capsulatus Reaction Centers with Asymmetric Pigment Content. J. Phys. Chem. B 121, 6989-7004 (2017).

48. P. Huppman, et al., Kinetics, Energetics, and Electronic Coupling of the Primary Electron Transfer Reactions in Mutated Reaction Centers of Blastochloris viridis. Biophysical Journal 82, 3186-3197 (2002).

49. C. Kirmaier, et al., Comparison of M-Side Electron Transfer in Rb. sphaeroides and Rb. capsulatus Reaction Centers. J. Phys. Chem. B 106, 1799-1808 (2002). 
50. J. Fajer, D. C. Borg, A. Forman, D. Dolphin, R. H. Felton, Anion Radical of Bacteriochlorophyll. Proceedings of the National Academy of Sciences 95, 2739-2741 (1973).

51. J. Fajer, D. C. Brune, M. S. Davis, A. Forman, L. D. Spaulding, Primary Charge Separation in Bacterial Photosynthesis: Oxidized Chlorophylls and Reduced Pheophytin. Proceedings of the National Academy of Sciences 72, 4956-4960 (1975).

52. A. A. Zabelin, A. M. Khristin, V. A. Shkuropatova, R. A. Khatypov, A. Ya. Shkuropatov, Primary electron transfer in Rhodobacter sphaeroides R-26 reaction centers under dehydration conditions. Biochimica et Biophysica Acta (BBA) - Bioenergetics 1861, 148238 (2020).

53. B. P. Paulson, J. R. Miller, W.-X. Gan, G. Closs, Superexchange and Sequential Mechanisms in Charge Transfer with a Mediating State between the Donor and Acceptor. J. Am. Chem. Soc. 127, 48604868 (2005).

54. T. Kondo, et al., Cryogenic Single-Molecule Spectroscopy of the Primary Electron Acceptor in the Photosynthetic Reaction Center. J. Phys. Chem. Lett. 11, 3980-3986 (2020).

55. H. Sumi, T. Kakitani, Unified Theory on Rates for Electron Transfer Mediated by a Midway Molecule, Bridging between Superexchange and Sequential Processes. J. Phys. Chem. B 105, 9603-9622 (2001).

56. K. Saito, H. Sumi, Unified expression for the rate constant of the bridged electron transfer derived by renormalization. J. Chem. Phys. 131, 134101 (2009).

57. H. Zhou, S. G. Boxer, Probing Excited-State Electron Transfer by Resonance Stark Spectroscopy. 1. Experimental Results for Photosynthetic Reaction Centers. J. Phys. Chem. B 102, 9139-9147 (1998).

58. H. Zhou, S. G. Boxer, Probing Excited-State Electron Transfer by Resonance Stark Spectroscopy. 2. Theory and Application. J. Phys. Chem. B 102, 9148-9160 (1998).

59. T. P. Treynor, S. G. Boxer, Probing Excited-State Electron Transfer by Resonance Stark Spectroscopy: 3. Theoretical Foundations and Practical Applications. J. Phys. Chem. B 108, 13513-13522 (2004).

60. T. P. Treynor, C. Yoshina-Ishii, S. G. Boxer, Probing Excited-State Electron Transfer by Resonance Stark Spectroscopy: 4. Mutations near $B_{L}$ in Photosynthetic Reaction Centers Perturb Multiple Factors that Affect $\mathrm{B}_{\mathrm{L}}{ }^{*} \rightarrow \mathrm{B}_{\mathrm{L}}{ }^{+} \mathrm{H}_{\mathrm{L}}^{-}$. J. Phys. Chem. B 108, 13523-13535 (2004).

61. K. Lao, L. J. Moore, H. Zhou, S. G. Boxer, Higher-Order Stark Spectroscopy: Polarizability of Photosynthetic Pigments. J. Phys. Chem. 99, 496-500 (1995).

62. S. G. Boxer, Stark Realities. J. Phys. Chem. B 113, 2972-2983 (2009).

63. M. Gouterman, "Optical Spectra and Electronic Structure of Porphyrins and Related Rings" in The Porphyrins, (Academic Press, 1978), pp. 1-165.

64. A. Warshel, P. Sharma, M. Kato, W. Parson, Modeling electrostatic effects in proteins. Biochimica et Biophysica Acta (BBA) - Proteins \& Proteomics 1764, 1647-1676 (2006). 
65. S. Schmidt, et al., Primary electron-transfer dynamics in modified bacterial reaction centers containing pheophytin-a instead of bacteriopheophytin-a. Spectrochimica Acta Part A: Molecular and Biomolecular Spectroscopy 51, 1565-1578 (1995).

66. C. Lauterwasser, U. Finkele, H. Scheer, W. Zinth, Temperature dependence of the primary electron transfer in photosynthetic reaction centers from Rhodobacter sphaeroides. Chemical Physics Letters 183, 471-477 (1991).

67. M. Saggu, N. M. Levinson, S. G. Boxer, Experimental Quantification of Electrostatics in $\mathrm{X}-\mathrm{H} \cdots \pi$ Hydrogen Bonds. J. Am. Chem. Soc. 134, 18986-18997 (2012).

68. J. M. Hughes, M. C. Hutter, J. R. Reimers, N. S. Hush, Modeling the Bacterial Photosynthetic Reaction Center. 4. The Structural, Electrochemical, and Hydrogen-Bonding Properties of 22 Mutants of Rhodobacter sphaeroides. J. Am. Chem. Soc. 123, 8550-8563 (2001).

69. J. M. Tharp, Y. S. Wang, Y. J. Lee, Y. Yang, W. R. Liu, Genetic incorporation of seven ortho-substituted phenylalanine derivatives. ACS Chem. Biol. 9, 884-90 (2014).

70. S. K. Jha, M. Ji, K. J. Gaffney, S. G. Boxer, Direct measurement of the protein response to an electrostatic perturbation that mimics the catalytic cycle in ketosteroid isomerase. PNAS 108, 16612-7 (2011).

71. L. Blankenburg, et al., Following local light-induced structure changes and dynamics of the photoreceptor PYP with the thiocyanate IR label. Physical Chemistry Chemical Physics 21, 66226634 (2019).

72. J. P. Allen, J. C. Williams, Relationship between the oxidation potential of the bacteriochlorophyll dimer and electron transfer in photosynthetic reaction centers. Journal of Bioenergetics and Biomembranes 27, 275-283 (1995).

73. W. J. Coleman, E. J. Bylina, D. C. Youvan, "Reconstitution of Photochemical Activity in Rhodobacter capsulatus Reaction Centers Containing Mutations at Tryptophan M-250 in the Primary Quinone Binding Site" in Current Research in Photosynthesis: Proceedings of the VIIIth International Conference on Photosynthesis Stockholm, Sweden, August 6-11, 1989, M. Baltscheffsky, Ed. (Springer Netherlands, 1990), pp. 149-152.

74. J. Wang, T. Ding, K. Wu, Coulomb Barrier for Sequential Two-Electron Transfer in a Nano-Engineered Photocatalyst. J. Am. Chem. Soc., jacs.0c06256 (2020).

75. G. E. M. Crisenza, D. Mazzarella, P. Melchiorre, Synthetic Methods Driven by the Photoactivity of Electron Donor-Acceptor Complexes. J. Am. Chem. Soc. 142, 5461-5476 (2020).

76. A. H. Proppe, et al., Bioinspiration in light harvesting and catalysis. Nature Reviews Materials, 1-19 (2020).

77. K. F. Biegasiewicz, et al., Photoexcitation of flavoenzymes enables a stereoselective radical cyclization. Science 364, 1166-1169 (2019). 
78. D. J. Heyes, et al., Photochemical Mechanism of Light-Driven Fatty Acid Photodecarboxylase. ACS Catal. 10, 6691-6696 (2020). 